\title{
Biomarcadores para la evaluación y diagnóstico del sindrome de ojo seco: una revisión
}

\author{
Biomarkers for the evaluation and diagnosis \\ of the dry eye syndrome: a review
}

\author{
Sara-Viviana Angulo-Sánchez ${ }^{1}$; David-Alejandro Ortiz-Avila ${ }^{1}$
}

Forma de citar: Angulo Sánchez SV, Ortiz Avila DA. Biomarcadores para la evaluación y diagnóstico del síndrome de ojo seco: una revisión. Salud UIS. 2020; 52(2): 89-99. doi: http://dx.doi.org/10.18273/revsal.v52n2-2020003 (c) (i)

\section{Resumen}

Introducción: El síndrome de ojo seco es una enfermedad en la que se generan signos y síntomas que conducen a alteraciones oculares prolongadas, por lo tanto, es relevante establecer con precisión la etiología de la enfermedad con la finalidad de establecer el tratamiento más efectivo, de allí, la importancia del desarrollo de exámenes innovadores como son los biomarcadores, los cuales permiten identificar con mayor precisión el cuadro clínico. Por esta razón, el presente trabajo pretende describir los principales avances de los biomarcadores de la superficie ocular y reconocer su aplicación clínica para el diagnóstico de ojo seco entre los años 2013 a 2018. Metodología: Se analizó literatura sobre biomarcadores empleados para el diagnóstico del ojo seco, mediante una revisión sistemática tipo narrativa de 2013 a 2018 por medio de los descriptores controlados "Dry Eye Syndrome" "biomarkers" "tear proteins" "eye proteins" seleccionados en DeCS y Pubmed; la búsqueda arrojó 48 estudios, de los cuales seleccionamos 21 para el análisis. Resultados: Son diversas las proteínas lagrimales que pueden ser relacionadas con la presencia y ausencia de la enfermedad, es vital que los biomarcadores sean valorados como una herramienta alternativa para diagnosticar con facilidad y precisión la enfermedad del ojo seco. Discusión: Los biomarcadores permiten reconocer los procesos patógenos y biológicos del síndrome de ojo seco, al reflejar el estado de la superficie ocular en presencia o ausencia de signos y síntomas, facilitando el diagnóstico precoz, seguimiento, tratamiento y control de la enfermedad.

Palabras clave: Enfermedad del ojo seco; Biomarcadores; Proteínas lagrimales; Proteínas oculares; Citocinas; metaloproteinasas de la matriz; Mucinas.

\begin{abstract}
Introduction: Dry eye syndrome is a disease in which signs and symptoms that lead to prolonged ocular alterations occur, therefore, it is relevant to accurately establish the etiology of the disease with the configuration of establishing the most effective treatment, hence the development of innovative exams such as biomarkers selected with greater precision the clinical picture. For this reason, the present work aims to describe the main advances of biomarkers of the ocular surface and to recognize their clinical application for the diagnosis of dry eye between 2013 and 2018. Metodology: Literature on biomarkers used for the diagnosis of dry eye was analyzed, by means of a systematic narrative review from 2013 to 2018 by means of the controlled descriptors "Dry Eye Syndrome" "biomarkers" "tear proteins" "eye proteins" selected in DeCS and Pubmed; The search yielded 48 studies and 21 studies were selected for the analysis. Results: There are several tear proteins that can be related to the presence and absence
\end{abstract}

1. Fundación Universitaria del Área Andina, Bogotá.

Correspondencia: Sara Viviana Angulo Dirección: Fundación Universitaria del Área Andina, Calle 69 No. 11a-56 Teléfono: +57-7421947 ext. 1823 Correo electrónico: sangulo8@areandina.edu.co 
of the disease, it is vital that biomarkers are evaluated as an alternative tool to easily and accurately diagnose dry eye disease. Discussion: Biomarkers allow to recognize the pathogenic and biological processes of dry eye syndrome, reflecting the state of the ocular surface in the presence or absence of signs and symptoms, facilitating early diagnosis, monitoring, treatment and control of the disease.

Keywords: Dry eye syndrome; Biomarkers; Tear proteins; Eye proteins; Cytokines; Matrix metalloproteinases, Mucins.

\section{Introducción}

El síndrome de ojo seco es una enfermedad cuyo número de casos ha ido aumentando en los últimos años, como resultado de una gran cantidad de factores que contribuyen a su aparición. Desde hace algunos años, el síndrome de ojo seco es considerado un problema de salud pública ${ }^{1}$ y corresponde a uno de los motivos de consulta más frecuentes para los profesionales de la salud visual. Es por ello, que en las últimas tres décadas, instituciones como el Tear Film and Ocular Surface Society (TIFOS) y el Dry Eye WorkShop (DEWS) han publicado resultados de sus investigaciones. En el año 2007, el DEWS dio a conocer la primera definición globalmente aceptada sobre ojo seco. Sin embargo, en el año 2017 a causa del trabajo mancomunado entre TFOS y DEWS II se redefinió el concepto de ojo seco como: "El síndrome de ojo seco es una enfermedad multifactorial de la superficie ocular caracterizada por una pérdida de la homeostasis de la película lagrimal, acompañada por síntomas oculares, en los cuales la inestabilidad e hiperosmolaridad de la película lagrimal, inflamación y daño de la superficie ocular, y anormalidades neurosensoriales juegan roles etiológicos"2.

Numerosos estudios han sido realizados hace más de una década con relación al síndrome de ojo seco, particularmente se han reportado que los factores de riesgo son fundamentales para comprender la enfermedad, dentro de los cuales se destacan: género femenino, edad ${ }^{3}$, hipocorreción del defecto refractivo ${ }^{4}$, cirugía refractiva, enfermedad tiroidea, nivel educativo ${ }^{5}$, enfermedad autoinmune ${ }^{6}$, uso de videoterminales ${ }^{7}$, cambios hormonales, factores ambientales ${ }^{8}$, cirugía de catarata ${ }^{9}$, exposición a radiación ionizante ${ }^{10}$, entre otros. Cifras obtenidas en investigaciones previas ${ }^{11,12,5}$, sugieren que entre el $17 \%$ al $21 \%$ de la población estudiada presenta la enfermedad de ojo seco. Por lo tanto, existen múltiples factores que pueden desencadenar la enfermedad, sin embargo, no existe un protocolo respecto al diagnóstico y/o tratamiento de la enfermedad, precisamente por la multifactorialidad de sus causas y el diverso comportamiento que puede presentar el cuadro clínico en condiciones similares.
Por lo anterior, el desarrollo de nuevas tecnologías es crucial para conducir a un diagnóstico y tratamiento asertivo ya que los signos y síntomas del paciente son poco específicos a la hora de distinguir entre varios tipos de la enfermedad ${ }^{12}$. Actualmente no hay una prueba "Gold Standar" disponible para diagnosticar la enfermedad ${ }^{13}$, usualmente deben aplicarse métodos clínicos tradicionales como lo son rompimiento de la película lagrimal, test de shirmer, lisamina verde, entre otras ${ }^{14}$, para posteriormente correlacionar los resultados; sin embargo, estas pruebas están sujetas a la colaboración del paciente y la destreza del examinador, lo cual puede resultar en falsos positivos o falsos negativos restando precisión al diagnóstico.

Por lo tanto, surgen los biomarcadores como un instrumento cuantificable y útil para identificar procesos patogénicos, procesos biológicos y la efectividad de tratamientos ${ }^{15}$, por ende, pueden ser empleados para prevenir, identificar o medir un riesgo ${ }^{16}$, así como diagnosticar, comprender mecanismos, evaluar la gravedad de una patología ${ }^{17}$ y monitorear la enfermedad $^{18}$; es decir que, ante la presencia, ausencia o concentración elevada en algún biomarcador se podrá detectar un estado patológico incluso antes que este inicie, o permitirá reconocer la efectividad de un tratamiento desde las primeras etapas de su aplicación. En consecuencia, un biomarcador reducirá la incertidumbre, al identificar las personas sanas de las enfermas ${ }^{19}$; en ese sentido, contará con sensibilidad y especificidad para detectar la enfermedad ${ }^{20}$.

Según el grupo de trabajo de definición de los Biomarcadores de los Institutos Nacionales de la Salud (1998), indicaron que los biomarcadores "miden y evalúan objetivamente como un indicador de procesos biológicos normales, procesos patogénicos o respuestas farmacológicas a una intervención terapéutica", éstos emplean técnicas con inmunoensayos y métodos bioquímicos para detectar proteínas secretas por la glándula lagrimal o generadas por células inflamatorias o epiteliales de la superficie ocular ${ }^{21}$. Por lo anterior, los biomarcadores son proteínas medibles en fluidos biológicos ${ }^{22}$ tales como sangre, orina, lagrimas, entre otras. Incluso los primeros estudios de muestras 
biológicas para diagnóstico de ojo seco datan que en 1990 "era pre-proteómica" donde reconocieron 10 proteínas lagrimale ${ }^{23}$ entre ellas: lactoferrina, lisozima, inmunoglobulina A secretora, glicoproteína Zinc alfa2, albúmina, inmunoglobulina $\mathrm{G}$, y la transferrina; quienes presentaron compromiso directo con el síndrome de ojo $\operatorname{seco}^{23}$. A partir de estos hallazgos, se fueron identificando más marcadores que podrían vincularse con la fisiopatogenia de la enfermedad ${ }^{24,25}$, de allí que, se han desarrollado diversas formas para cuantificar y categorizar las proteínas de la superficie ocular ${ }^{26}$.

De este modo, los biomarcadores constituyen una herramienta alternativa para diagnosticar el síndrome de ojo seco, la ventaja es que puede emplear muestras de lágrima o conjuntiva para evaluar el tejido ocular; permitiendo explicar con mayor precisión los subtipos de la enfermedad ${ }^{13}$, consiguiendo que el tratamiento sea cada vez más específico a cada paciente $^{12}$, al evaluar la respuesta al tratamiento desde su aplicación. Sin embargo, su viabilidad ha sido cuestionada debido al difícil acceso, elevados $\operatorname{costos}^{27}$ y el requerimiento de personal calificado para la toma y análisis de las muestras para cada procedimiento ${ }^{28}$. No obstante, los biomarcadores constituyen métodos diagnósticos alternativos que evalúan la superficie ocular permitiendo detectar con precisión la presencia o ausencia del síndrome del ojo seco, lo que podría afectar positivamente en la calidad de vida del paciente. Por esta razón, la presente revisión tiene como objetivo describir los principales avances de los biomarcadores de la superficie ocular y reconocer su aplicación clínica para el diagnóstico de ojo seco entre los años 2013 a 2018.

\section{Metodología}

Se realizó una revisión sistemática tipo narrativa para describir el estado del arte, desde una perspectiva teórica sobre los biomarcadores de la superficie ocular empleados para el diagnóstico de la enfermedad del ojo seco. La búsqueda se llevó a cabo en las bases de datos Science Direct, Pubmed, Proquest y Scopus, limitándola a artículos que fueron publicados entre 2013 y 2018. La estrategia de búsqueda empleó descriptores controlados: "Dry Eye Syndrome" "biomarkers" "tear proteins" "eye proteins" seleccionados en DeCS y Pubmed, de igual manera se utilizaron operadores boolanos AND, OR y NOT.

La revisión incluyó publicaciones primarias y secundarias en idioma español e inglés, entre los años 2013 a 2018, se aprobaron los diseños de investigación descriptivos, semiexperimentales y experimentales, artículos con uno o más de los descriptores mencionados los cuales fueron revisados en el título, resumen, palabras clave o introducción. Se excluyeron estudios que mencionaran en el cuerpo del trabajo métodos tales como citología de impresión, osmolaridad, meiboscopia y métodos tradicionales (schirmer I y II, tinción con fluoresceína, Break Up Time, tinción con lisamina verde, hilo rojo fenol), de igual manera se excluyeron artículos de revisión de literatura y metaanálisis.

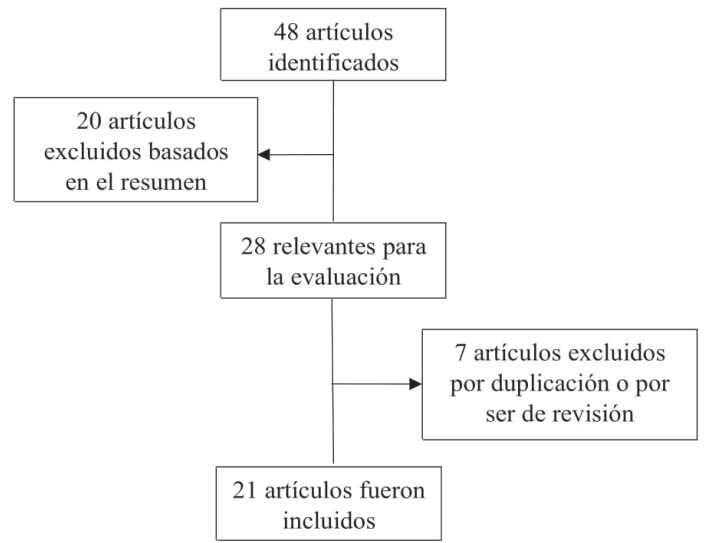

Figura 1. Flujograma de la búsqueda y selección de los artículos incluidos en la revisión sistemática tipo narrativa.

Los resúmenes de los artículos, que inicialmente se reconocieron como potencialmente relevantes al estudio fueron evaluados por los investigadores, identificando si cumplían con los criterios de inclusión mencionados anteriormente, para ello, cada artículo fue revisado exhaustivamente y se procedió a clasificar la información en una matriz con el programa Excel, resaltando título, autor, año, propósito del estudio, proteína evaluada, aporte clínico y comentarios de los investigadores.

La información recolectada fue analizada mediante discusiones clínicas entre los investigadores, tal discusión incluyó la síntesis de la información por medio del reconocimiento de las proteínas comúnmente presentes en el síndrome de ojo seco activo y en el control de la enfermedad, se clasificaron las proteínas según las capas de la película lagrimal (capa mucina, capa acuosa y capa lipídica) y se identificaron otros aportes clínicos para realizar categorías amplias de la información.

\section{Resultados}

Con base en la evidencia encontrada se construyó la siguiente tabla, la cual reúne el nombre del biomarcador, su peso molecular, función molecular y los principales aportes clínicos en relación con el diagnóstico del síndrome de ojo seco. 


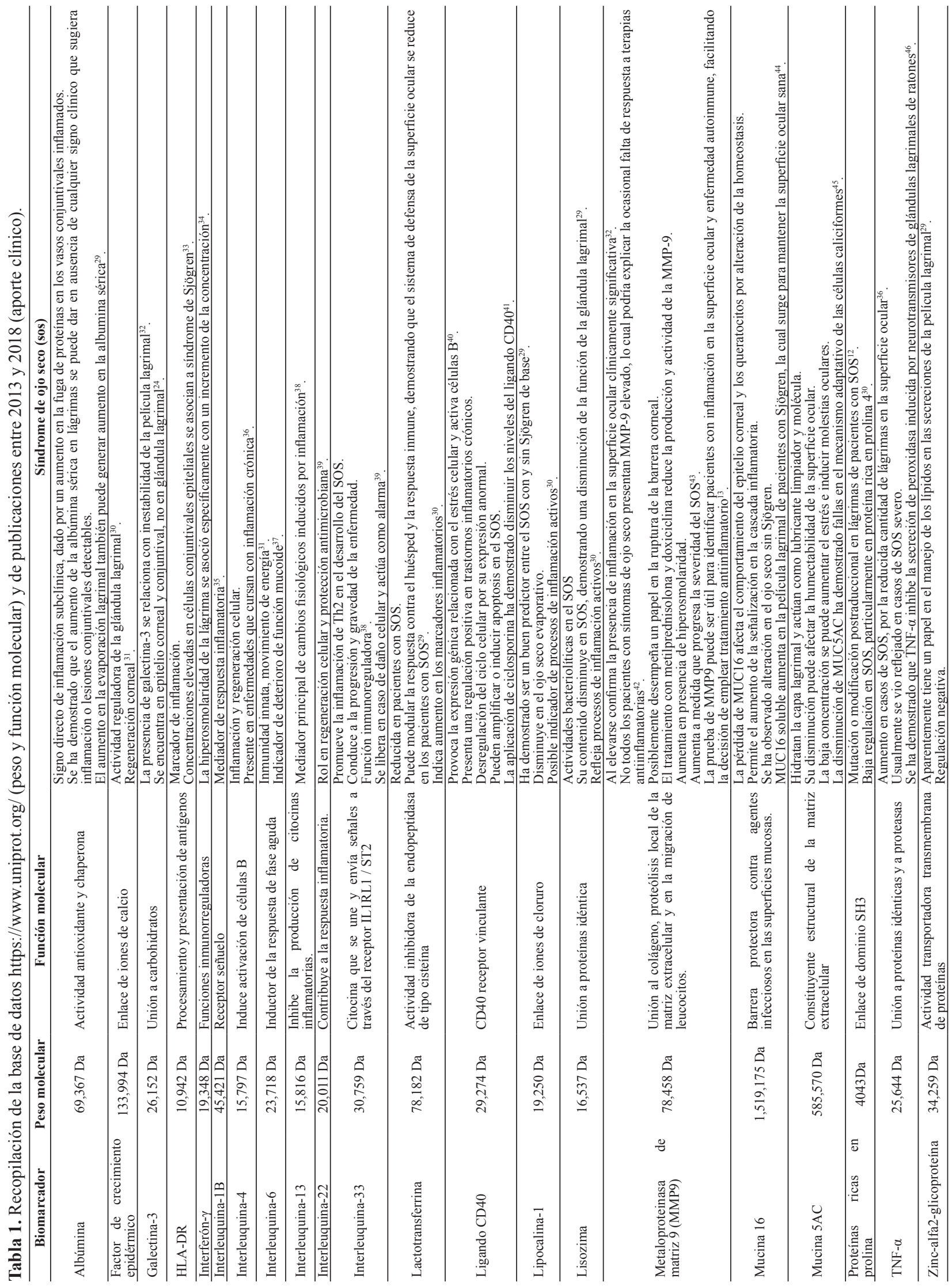




\section{Discusión}

Desde que empezaron a ser estudiados, los biomarcadores constituyeron un instrumento cuantificable y útil para identificar procesos patogénicos, procesos biológicos y la efectividad de un tratamiento ${ }^{15}$, por lo tanto, podían ser empleados para prevenir, identificar o medir un riesgo ${ }^{16}$, así como diagnosticar, comprender mecanismos, evaluar la gravedad de una patología ${ }^{17}$ y monitorear la enfermedad $^{18}$; es decir, que ante la presencia, ausencia o concentración elevada de algún biomarcador se podrá detectar un estado patológico incluso antes que éste inicie, o permitirá reconocer la efectividad de un tratamiento desde las primeras etapas de su aplicación. En consecuencia, un biomarcador reducirá la incertidumbre de una patología al identificar las pruebas diagnósticas que le permitirán detectar una condición clínica, para separar adecuadamente las personas sanas de las enfermas ${ }^{19}$; en ese sentido, el biomarcador tendrá sensibilidad y especificidad para detectar una enfermedad ${ }^{20}$.

Del mismo modo, los biomarcadores deben ser fácilmente evaluados en la práctica clínica; estos corresponden a proteínas medibles en fluidos biológicos ${ }^{22}$ tales como sangre, orina, lagrima, entre otras. En el ámbito de la superficie ocular, los biomarcadores constituyen una herramienta alternativa para diagnosticar las enfermedades de la superficie ocular, tal como ocurre con el síndrome de ojo seco, la ventaja de este fluido es que puede ser fácilmente recogido, y puede emplear muestras de lágrima o conjuntiva para evaluar el tejido ocular; en consecuencia, gracias a su surgimiento se ha logrado explicar con mayor precisión los subtipos de la enfermedad ${ }^{29}$, y ha conseguido que el tratamiento sea cada vez más específico a cada paciente ${ }^{12}$, permitiendo evaluar la respuesta al tratamiento desde su aplicación. Sin embargo, su viabilidad ha sido cuestionada debido a su difícil acceso, elevados $\operatorname{costos}^{27}$ y el requerimiento de personal sumamente calificado para la toma y análisis de las muestras para cada procedimiento ${ }^{28}$.

Los primeros estudios de muestras biológicas para diagnóstico de ojo seco datan que en 1990, se denominó la "era pre-proteómica"; sin embargo, debido a las limitadas técnicas de la época, tan solo un poco más de 10 proteínas lagrimales fueron reconocidas de manera confiable ${ }^{29}$, entre ellas están: lactoferrina, lisozima, inmunoglobulina A secretora, glicoproteína Zinc alfa2, albúmina, inmunoglobulina $\mathrm{G}$, y la transferrina; a quienes se les vio compromiso directo con el síndrome de ojo $\operatorname{seco}^{23}$. A partir de estos hallazgos, se fueron identificando más marcadores que podrían vincularse de uno u otra forma con la fisiopatogenia de la enfermedad ${ }^{24,25}$, de manera que, se fueron desarrollando diversas formas para cuantificar y categorizar las proteínas de la superficie ocular ${ }^{26}$.

Actualmente, persiste la dificultad para diagnosticar y controlar pacientes con el síndrome de ojo seco debido a la variabilidad de los síntomas, ausencia de pruebas altamente confiables, débiles correlaciones entre pruebas clínicas y, por tanto, afectación en la calidad de vida de los pacientes ${ }^{17}$, por ende, se hace evidente el valor clínico de desarrollar biomarcadores específicos para el diagnóstico. Incluso, se ha demostrado que la lagrima tiene una compleja composición de proteínas, lípidos, mucinas, agua y sales, de las cuales han sido identificadas 1526 proteínas gracias a los análisis proteómicos, éstas se consideran menos complejas que el fluido plasmático o suero ${ }^{20}$. Por lo tanto, los biomarcadores son indicadores vitales que permiten el estudio de la actividad de la enfermedad y la toma de decisiones.

Por lo tanto, la evidencia científica respalda el análisis del proteoma lagrimal como prometedor, dado que permite ampliar el conocimiento con relación al síndrome de ojo seco, puesto que cambios en las proteínas lagrimales posiblemente pueden reflejar el estado de la superficie ocular y de la enfermedad ${ }^{29}$, es decir, ayudarían con el proceso de diagnóstico precoz, seguimiento de la enfermedad y opciones de tratamiento ${ }^{30}$. No obstante, los procedimientos en relación con los biomarcadores lagrimales en la actividad clínica aún están en progreso. Por su parte, autores como Bohem ${ }^{12}$ sugieren que no se deben tomar los perfiles de proteínas como una alternativa diagnóstica sin considerar los diferentes aspectos patológicos o etiológicos de la enfermedad del ojo seco. Por esta razón, es relevante investigar la información que proporcionan los biomarcadores acerca del daño ocular, ya que tienen la capacidad de diagnosticar el impacto de la disfunción lagrimal en la superficie ocular ${ }^{21}$, evidenciando signos de inflamación precoz y permitiendo la valoración temprana de aquellos casos donde las pruebas de tinción tradicional no presentan daño evidente en la superficie, aunque exista reporte de síntomas.

Con respecto a la lisozima, es la primera proteína lagrimal reconocida ${ }^{47,48}$, se encuentra reducida en pacientes con ojo seco idiopático y con síndrome de Sjögren en comparación con los controles; mientras que otros autores ${ }^{49}$ indican que su concentración no difiere en ojos secos por síndrome de Sjögren ni en síndromes de ojo seco no relacionados al Sjögren versus los 
controles. Sobre la lipocalina, han indicado que es menor en pacientes con ojo seco síndrome de Sjögren, en pacientes con ojo seco temprano hiperevaportivo pero no en pacientes con ojo seco no Sjögren ${ }^{49}$, así mismo ha presentado una correlación inversa con el rompimiento de la película lagrimal ${ }^{50}$.

Por su parte, la lactoferrina (transferrina) es uno de los componentes más abundantes en la película lagrimal sana, contribuye a los mecanismos de retención de hierro contra patógenos ${ }^{49}$ y mitiga el estrés oxidativo ${ }^{51}$; mientras que, la albúmina sérica presenta uno de los contenidos más bajos en las proteínas lagrimales; sin embargo ambas derivan del plasma, por medio de una filtración pasiva en los $\operatorname{vasos}^{52}$ en pacientes con lágrimas sanas, no obstante, la albúmina es un indicador de la inflamación subclínica debido a la filtración de vasos conjuntivales inflamados ${ }^{53}$. En un estudio de ciento sesenta pacientes con sospecha de ojo seco de leve a moderado, en el 2013 se encontró un aumento significativo en la albúmina en ausencia de signos clínicos o lesiones conjuntivales, lo que indicaría que la albúmina es un indicador objetivo para evaluar los estados inflamatorios de la superficie ocular cuando no son evidentes los síntomas; mientras que el mismo estudio presentó una disminución significativa en la transferrina en pacientes con ojo seco temprano frente a los controles ${ }^{29}$, por lo tanto, al existir una disminución en la transferrina ocurrirá un desequilibrio de hierro en las lágrimas conduciendo al daño celular por la no unión con el hierro ${ }^{54}$, disminución que también se ha visto en pacientes con ojo seco acuodeficiente ${ }^{55}$.

La alteración de la galectina-3 ha sido estudiada predominantemente en pacientes con cáncer y enfermedad inflamatoria, pero comúnmente se observa en estados patológicos de síndrome de ojo $\mathrm{seco}^{56}$, se ha especulado que la galectina-3 puede propagarse en las lágrimas de pacientes con ojo seco como consecuencia de la permeabilidad vascular y conjuntival ${ }^{32}$; estructuras incompletas de galectina-3 en casos patológicos inhiben una variedad de procesos tales como el crecimiento tumoral y la curación de heridas ${ }^{57}$.

Con respecto a las interleuquinas presentan un rol importante en la respuesta inmune adaptativa, ya que son indispensables para la propagación de la inflamación ${ }^{58}$, se ha demostrado especialmente que IL- $1 \beta$, se exacerba en ojos con deficiencia acuosa $a^{59}$ y se aumenta en la producción y activación proinflamatoria ${ }^{60}$. por su parte la IL-17 aumenta su concentración en pacientes con ojo seco, probablemente jugando un papel importante en los procesos de inflamación de la superficie ocular ${ }^{36}$; adicionalmente, se ha correlacionado la puntuación de tinción con fluoresceína corneal ${ }^{61}$ y conjuntival $^{62}$, con respecto a elevadas concentraciones de IL-17 sérico en pacientes con ojo seco por enfermedad inflamatoria sistémica. La presencia de IL-17 estimula la producción de MMP-9 y causa daño en el epitelio corneal $^{38}$, también se ha demostrado asociación entre la presencia de IL-6 y la producción de MMP-9 ${ }^{23}$. Por otro lado, se ha encontrado que en los pacientes con síndrome de ojo seco con Sjögren se compromete la IL-17 debido a la reacción inmunitaria sistémica A local, mientras que los ojo seco no Sjögren pueden ocurrir debido a situaciones estresantes, como la desecación de la superficie, lo que puede conducir a aumentos de las citoquinas IL- $6^{36}$, quien lidera en la resolución de la inmunidad innata aguda y direcciona hacia una respuesta inmune adquirida; así mismo existe aumento de la IL- $33^{63}$ en pacientes con ojos secos. En consecuencia, la diferenciación de los subtipos de ojo seco es crucial ya que algunas opciones terapéuticas se enfocan en suplementar el componente faltante de la película lagrimal, mientras que algunas opciones más recientes se orientan hacia la utilización de moléculas de tipo antiinflamatorias ${ }^{64}$.

Frente al factor de necrosis tumoral- $\alpha$ (TNF- $\alpha)$ e interferón- $\gamma$ (IFN- $\gamma$ ), son citocinas presentes en la respuesta aguda que mejoran las respuestas inmunes celulares $^{65}$; sin embargo, el inconveniente principal para usar citoquinas lagrimales como marcadores, es que no existen estudios clínicos de tipo longitudinal ${ }^{66}$, lo que limita la confianza en el uso. Por otro lado, se ha indicado que el TNF- $\alpha$ aumenta en la producción $\mathrm{y}$ activación proinflamatoria ${ }^{60}$, mientras que el IFN- $\gamma$ aparentemente presenta un papel importante en la patogénesis del ojo $\operatorname{seco}^{67}$, ya que esta causa apoptosis y pérdida de células de Goblet conjuntivales ${ }^{38}$, así, ante niveles aumentados de IFN- $\gamma$, el tratamiento requerido será inmunomoduladores de células $\mathrm{T}$, tal como la ciclosporina $\mathrm{A}^{67}$.

Por otro lado, las MMP-9 son un predictor de la severidad clínica de la enfermedad y se le considera un marcador clínico para monitorear pacientes ${ }^{68}$, de igual manera, se ha indicado que pacientes con ojo seco secundario a otras enfermedades presentan una elevada actividad de MMP- ${ }^{70}$, adicionalmente aumenta en condiciones hiperosmolares y contribuye a la interrupción de la barrera corneal, incrementando sus niveles a medida que se eleva la severidad del ojo seco $^{13}$. Una de las alternativas más específicas para medir las MMP-9 es la prueba InflammaDry que con ayuda de una solución reguladora y en un lapso muy corto, es capaz de detectar niveles anormalmente elevados de MMP-9 (40ng/ml o mayor) en pacientes con ojo seco ${ }^{70}$. 
La diferenciación entre cada subtipo de la enfermedad facilita el diagnóstico y tratamiento, así la medida de la mucina MUC5AC quien es secretada por las células caliciformes conjuntivales, se encuentra disminuida en el ojo seco perjudicando la humectabilidad del epitelio, dada la naturaleza hidrofílica de las mucinas que resultan de una pesada glicosilación que ayuda a mantener el fluido de la superficie ${ }^{71}$, debido a que la MUC5AC tiene propiedades reológicas que ayuda a contener líquidos y lubrican la superficie epitelial ${ }^{71}$, incluso hay reportes ${ }^{73,7}$ que indican la relación entre el uso de videoterminales con el desarrollo del síndrome de ojo seco, debido a la disminución en la concentración de la MUC5AC en ausencia de hiperemia o trastornos epiteliales. Asimismo, se ha indicado que las mucinas se encuentran afectadas en pacientes con deficiencia acuosa $^{74,59}$. Por su parte, Shirai, et al. ${ }^{44}$, concluyen que algunas mucinas como la MUC16 juegan un rol importante en la homeostasis conjuntival y corneal, así las cosas, la pérdida de MUC16 puede conducir a una reacción inflamatoria subclínica en ausencia de estímulos externos adicionales.

A continuación, se presenta una figura que resumen las proteínas comúnmente afectadas en el ojo seco, para ello se tomó en cuenta el nombre de la proteína y su información funcional.

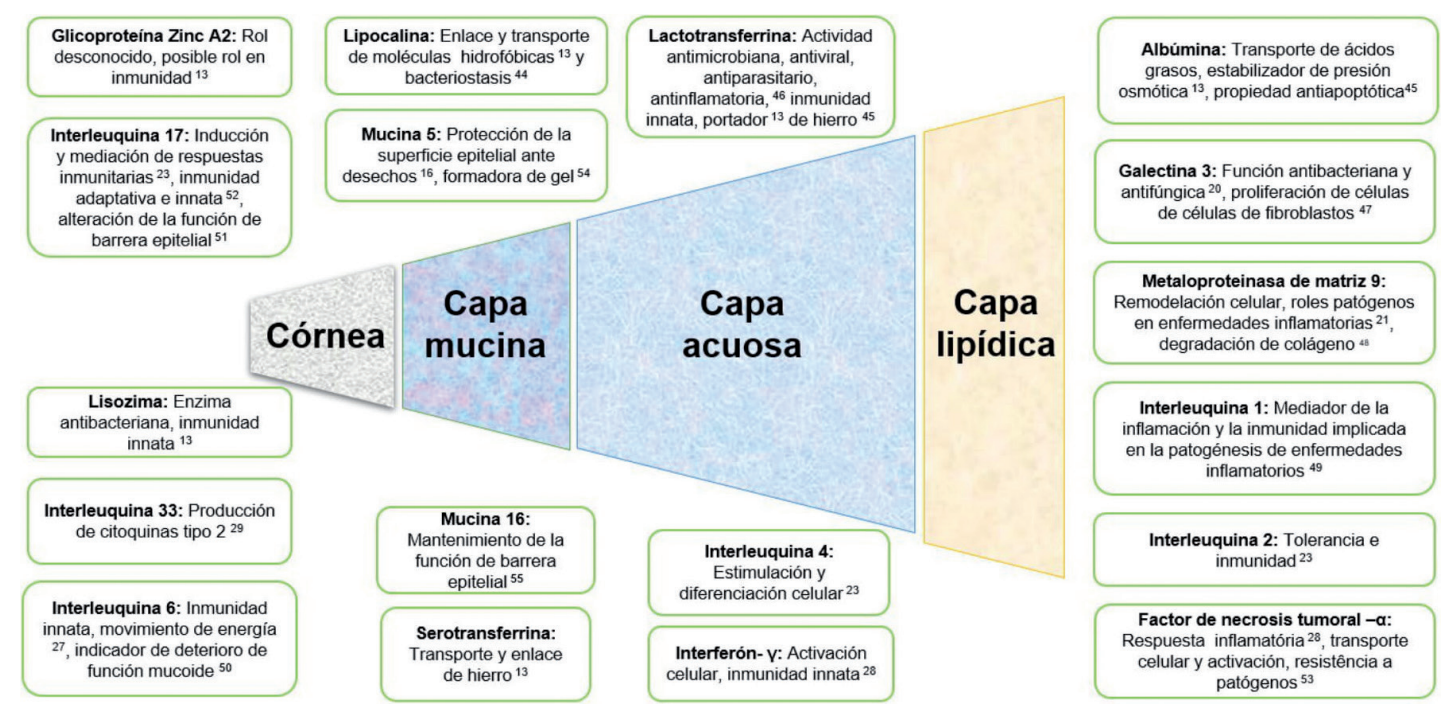

Figura 2. Resumen de las proteínas lagrimales reconocidas según su función, elaborado por autores.

Por consiguiente, aunque son evidentes los progresos que ha proporcionado el reconocimiento de biomarcadores de superficie ocular, existe una serie de limitaciones que pueden restringir su uso en la práctica clínica, por ejemplo, las diferencias en la recolección de la lágrima, lo que puede conducir a resultados diferentes $^{75}$. Igualmente, durante la recolección es necesario evitar la mínima estimulación de la película lagrimal, dado que si existe una ruptura lagrimal probablemente aparecerán proteínas lagrimales de tipo refleja ${ }^{68}$ lo que conduciría a análisis y resultados diferentes; por ejemplo, las concentraciones de IL-1 pueden alterarse en el ojo seco con lágrima basal pero no en la refleja ${ }^{69}$; en consecuencia, es evidente la necesidad de estandarización para la toma de tests clínicos en futuras investigaciones. En este orden de ideas, los análisis proteómicos son prometedores para obtener mayor información sobre la patogénesis de la enfermedad, el control de agentes farmacológicos y el diseño de herramientas diagnósticas ${ }^{30}$. Es conveniente resaltar que la importancia de los biomarcadores no solo se limita a enfermedades de la superficie ocular, sino que también cuentan con utilidad clínica en enfermedades sistémicas como la orbitopatía tiroidea ${ }^{36}$, diabetes mellitus ${ }^{76} \mathrm{y}$ el cáncer ${ }^{77}$, lo que podría ayudar a comprender o controlar la progresión de la enfermedad.

\section{Conclusiones}

La investigación sobre los biomarcadores es un área prometedora y necesaria en términos de diagnóstico, evaluación, tratamiento y control de la enfermedad; los modelos actuales sugieren la necesidad de ahondar en tecnologías que midan la película lagrimal como herramienta clave en la fisiopatogenia del ojo seco, así como la estandarización de procesos para su aplicación. Se observó que el síndrome de ojo seco puede cambiar patrones del proteoma lagrimal, lo cual justifica la 
necesidad de profundizar en procesos patológicos completos que permitan obtener una información detallada sobre las proteínas que se afectan durante el proceso. Sin embargo, es importante destacar que aun la función biológica real de los diferentes biomarcadores de la película lagrimal no está plenamente comprendida, a pesar de ello, estos proporcionan una información in vivo del estado celular, lo cual promueve el conocimiento de los procesos de inflamación característicos del síndrome. Por lo anterior, es relevante comprender el desarrollo del síndrome de ojo seco, ya que hoy en día representa un problema para la salud pública, puesto que ocasiona incomodidad ocular, fatiga y trastornos visuales que pueden comprometer las actividades diarias de una persona. Por ello, la identificación temprana de los subtipos y el control de la severidad es crucial para mejorar el bienestar del paciente.

En este orden de ideas, el presente trabajo evidenció que son varias las investigaciones que sustentan el estudio de los perfiles proteómicos lagrimales como una herramienta valiosa y consistente, con capacidad para proporcionar información en casos donde la sintomatología puede considerarse "normal" tomando como referencia las pruebas tradicionales; mientras que los biomarcadores pueden ofrecer un diagnóstico precoz de la enfermedad, en tanto que, permite mitigar las consecuencias a largo plazo, ya que son una herramienta que identifica la presencia o ausencia de inflamación en la superficie ocular, siendo una guía para la toma de decisiones terapéuticas. En consecuencia, este trabajo permite informar al profesional de la salud visual sobre los principales proteomas que se han vinculado con el diagnóstico del síndrome de ojo seco, lo cual constituye un elemento clave a la hora de evaluar en el cuadro clínico del paciente; de igual manera la presente revisión pretende suscitar la necesidad de indagar en el tema, dada la ausente información disponible en poblaciones latinoamericanas con síndrome de ojo seco, principalmente para el aprovechamiento del valor diagnóstico de los biomarcadores en la superficie ocular en estas poblaciones.

\section{Referencias}

1. Bhavsar AS, Bhavsar SG, Jain SM. A review on recent advances in dry eye: Pathogenesis and management. Oman J Ophthalmol. 2011; 4(2): 5056. doi: 10.4103/0974-620X.83653.

2. Craig JP, Nelso JD, Azar DT, Belmonte C, Bron AJ, Chauhan S $\mathrm{K}$, et al. TFOS DEWS II Report executive summary. Ocul Surf. 2017; 15(4): 802-812. doi: 10.1016/j. jtos.2017.08.003.

3. Ahn JM, Lee SH, Rim THT, Park RJ, Yang HS, Kim T, et al. Prevalence of and risk factors associated with dry eye: The Korea national health and nutrition examination survey 2010-2011. Am J Ophthalmol. 2014; 158(6): 1205-1214.e7. doi: 10.1016/j.ajo.2014.08.021.

4. Jie Y, Xu L, Wu YY, Jonas JB. Prevalence of dry eye among adult Chinese in the Beijing eye study. Eye (Lond). 2008; 23(3), 688-693. doi: 10.1038/ sj.eye. 6703101.

5. Galor A, Feuer W, Lee DJ, Flórez H, Carter D, Bozorgmehr P, et al. Prevalence and risk factors of dry eye syndrome in a United States Veterans affairs population. Am J Ophthalmol. 2011; 152(3): 377 384.e2. doi: 10.1016/j.ajo.2011.02.026.

6. López-Rubio S, de Alba-Castilla MA, RodríguezGarcía A. Prevalencia de manifestaciones oftalmológicas en pacientes con lupus eritematoso sistémico. Rev Mex Oftalmol. 2012; 86(4): 240-249.

7. Uchino M, Yokoi N, Uchino Y, Dogur M, Kawashima M, Komuro A, et al. Prevalence of dry eye disease and its risk factors in visual display terminal users: the Osaka Study. Am J Ophthalmol. 2013; 156(4): 759-733. doi: 10.1016/j.ajo.2013.05.040.

8. Ginés JC. Síndrome del ojo seco. Rev Paraguay Reumatol. 2015; 1(1): 49-55.

9. Cho YK, Kim MS. Dry eye after cataract surgery and associated intraoperative risk factors. Korean J Ophthalmol. 2009; 23(2): 65-73. doi: 10.3341/ kjo.2009.23.2.65.

10. Özkurt H, Özkurt YB, Basak M. Is dry eye syndrome a work-related disease among radiologists? Diagn Interv Radiol. 2006; 12(4): 163-165.

11. Liu NN, Liu L, Li J, Sun YZ. Prevalence of and risk factors for dry eye symptom in mainland china: a systematic review and meta-analysis. J Ophthalmol. 2014; 2014: 748654. doi: 10.1155/2014/748654.

12. Boehm N, Funke S, Wiegand M, Wehrwein N, Pfeiffer N, Grus FH. Alterations in the tear proteome of dry eye patients--a matter of the clinical phenotype. Invest. Ophthalmol Vis Sci. 2013; 54(3): 2385-2392. doi: 10.1167/iovs.11-8751.

13. Lanzini M, Curcio C, Colabelli-Gisoldi RA, Mastropasqua A, Calienno R, Agnifili L, et al. In vivo and impression cytology study on the effect of compatible solutes eye drops on the ocular surface epithelial cell quality in dry eye patients. Mediators Inflamm. 2015; 2015: 351424. doi: $10.1155 / 2015 / 351424$.

14. Schein O, Tielsch JM, Munõz B, Bandeen-Roche $\mathrm{K}$, West S. Relation between signs and symptoms of dry eye in the elderly: a population-based perspective. 
Ophthalmology. 1997; 104(9): 1395-1401.

15. Strimbu K, Tavel JA. What are biomarkers?. Curr Opin HIV AIDS. 2010; 5(6): 463-466. doi: 10.1097/ COH.0b013e32833ed177.

16. Mayeux R. Biomarkers: potential uses and limitations. NeuroRx. 2004; 1(2): 182-188. doi: 10.1602/neurorx.1.2.182.

17. Deschamps N, Baudouin C. Dry Eye and Biomarkers: present and future. Curr Ophthalmol Rep. 2013; 1: 65-74.

18. Pentyala S, Muller J, Tumillo T, Roy A, Mysore P, Pentyala S. A novel point-of-care biomarker recognition method: validation by detecting marker for diabetic nephropathy. Diagnostics (Basel). 2015; 5(2): 177-188. doi: 10.3390/diagnostics5020177.

19. Medina M. Generalidades de las pruebas diagnósticas, y su utilidad en la toma de decisiones médicas". Rev Colomb Psiquiatr. 2011; 40(4): 787-797.

20. Hagan S, Martin E, Enríquez-de-Salamanca A. Tear fluid biomarkers in ocular and systemic disease: potential use for predictive, preventive and personalised medicine. EPMA J. 2016; 7(1):15.

21. Foulks GN, Pflugfelder SC. New testing options for diagnosing and grading dry eye disease. Am J Ophthalmol. 2014; 157(6): 1122-1129. doi: 10.1016/j.ajo.2014.03.002.

22. Bökenkamp A, Franke I, Schlieber M, Düker G, Schmitt J, Buderus S, et al. Beta-trace protein--a marker of kidney function in children: Original research communication-clinical investigation. Clin Biochem. 2007; 40(13-14): 969-975. doi: 10.1016/j. clinbiochem.2007.05.003.

23. Zhou L, Beuerman RW. Tear analysis in ocular surface diseases. Prog Retin Eye Res. 2012; 31(6): 527-550. doi: 10.1016/j.preteyeres.2012.06.002.

24. Mii S, Nakamura K, Takeo K, Kurimoto S. Analysis of human tear proteins by two-dimensional electrophoresis. Electrophoresis. 1992; 13(1): 379382. doi: 10.1002/elps.1150130177.

25. Glasson M, Molloy M, Walsh B, Willcox M, Morris C, Williams K. Development of mini-gel technology in two-dimensional electrophoresis for mass-screening of samples: application to tears. Electrophoresis. 1998; 19(5): 852-855. doi: 10.1002/elps.1150190541.

26. Baier G, Wollensak G, Mur E, Redl B, Stoffler G, Gottinger W. Analysis of human tear proteins by different high-performance liquid chromatographic techniques. J Chromatography. 1990; 525: 319-328. doi: https://doi.org/10.1016/S0378-4347(00)83408-8.

27. Zeev MS, Miller DD, Latkany R. Diagnosis of dry eye disease and emerging technologies. Clin
Ophthalmol. 2014; 8: 581-590. doi: 10.2147/OPTH. S45444.

28. Amur S, LaVange L, Zineh I, Buckman-Garner S, Woodcock J. Biomarker Qualification: Toward a multiple stakeholder framework for biomarker development, regulatory acceptance, and utilization. Clin Pharmacol Ther. 2015; 98(1): 34-46. doi: 10.1002/cpt.136.

29. Versura P, Bavelloni A, Grillini M, Fresina M, Campos EC. Diagnostic performance of a tear protein panel in early dry eye. Mol Vis. 2013; 19: 1247-1257.

30. Hohenstein-Blaul NT, Funke S, Grus FH. Tears as a source of biomarkers for ocular and systemic diseases. Exp Eye Res. 2013; 117: 126-137. doi: 10.1016/j.exer.2013.07.015.

31. Pinto-Fraga J, Enríquez-de-Salamanca A, Calonge M, González-García MJ, López-Miguel A, Lópezde la Rosa A. Severity, therapeutic, and activity tear biomarkers in dry eye disease: An analysis from a phase III clinical trial. Ocul Surf. 2018; 16(3): 368376. doi: 10.1016/j.jtos.2018.05.001.

32. Uchino Y, Mauris J, Woodward AM, Dieckow J, Amparo F, Dana R, et al. Alteration of galectin-3 in tears of patients with dry eye disease. Am J Ophthalmol. 2015; 159(6): 1027-1035.e3. doi: 10.1016/j.ajo.2015.02.008.

33. Brignole F, Riancho L, Ismail D, Deniaud M, Amrane M, Baudouin C. Correlation between the inflammatory marker HLA-DR and signs and symptoms in moderate to severe dry eye disease. Invest Ophthalmol Vis Sci. 2017; 58(4): 2438-2448. doi: 10.1167/iovs.15-16555.

34. Jackson DC, Zeng W, Wong CY, Mifsud EJ, Williamson NA, Ang C, et al. Tear interferongamma as a biomarker for evaporative dry eye disease. Invest Ophthalmol Vis Sci. 2016; 57(11): 4824-4830. doi: 10.1167/iovs.16-19757.

35. Grosskreutz CL, Hockey HU, Serra D, Dryja TP. Dry eye signs and symptoms persist during systemic neutralization of IL-1 $\beta$ by Canakinumab or IL-17A by Secukinumab. Cornea. 2015; 34(12): 1551-1556. doi: 10.1097/ICO.0000000000000627.

36. Lee S, Jung S, Min S, Chul S, Min S, Kim Ti, et al. analysis of tear cytokines and clinical correlations in Sjögren Syndrome dry eye patients and non-Sjogren syndrome dry eye patients. Am J Ophthalmol. 2013; 156(2): 247-253.e1. doi: 10.1016/j.ajo.2013.04.003.

37. Dogru MW, Ibrahim OS, Matsumoto Y, Ogawa J, Shimazaki J, Tsubota K. The effects of 2 weeks senofilcon-A silicone hydrogel contact lens daily wear on tear functions and ocular surface health status. Cont Lens Anterior Eye. 2011; 38(6): 435- 
441. doi: 10.1016/j.clae.2010.12.001.

38. Pflugfelder S, Corrales R, de Paiva C. T helper cytokines in dry eye disease. Exp Eye Res. 2013; 117: 118-125. doi: 10.1016/j.exer.2013.08.013.

39. Luo G, Xin Y, Qin D, Yan A, Zhou Z, Liu Z. Correlation of interleukin-33 with Th cytokines and clinical severity of dry eye disease. Indian J Ophthalmol. 2018; 66(1): 39-43. doi: 10.4103/ijo. IJO_405_17.

40. Tan X, Sun S, Liu Y, Zhu T, Wang K, Ren T, et al. Analysis of Th17-associated cytokines in tears of patients with dry eye syndrome. Eye (Lond). 2014; 28(5): 608-613. doi: 10.1038/eye.2014.38.

41. Jobling K, Fai W. CD40 as a therapeutic target in Sjögren's syndrome. Expert Rev Clin Immunol. 2018; 24(2): 121-132. doi: 10.1080/1744666X.2018.1485492.

42. Lanza NL, McClellan AL, Batawi H, et al. Dry eye profiles in patients with a positive elevated surface matrix metalloproteinase 9 point-of-care test versus negative patients. Ocul Surf. 2016; 14(2): 216-223. doi: 10.1016/j.jtos.2015.12.007.

43. Sambursky R. Presence or absence of ocular surface inflammation directs clinical and therapeutic management of dry eye. Clin Ophthalmol. 2016; 10: 2337-2343. doi: 10.2147/OPTH.S121256.

44. Shirai K, Okada Y, Cheon DJ, Miyajima M, Behringer RR, Yamanaka O, et al. Effects of the loss of conjunctival Muc16 on corneal epithelium and stroma in mice. Invest Ophthalmol Vis Sci. 2014; 55(6): 3626-3637. doi: 10.1167/iovs.13-12955.

45. Uchino Y, Uchino M, Yokoi N, Dogru M, Kawashima $\mathrm{M}$, Okada N, et al. Alteration of tear mucin 5ac in office workers using visual display terminals: The Osaka Study. JAMA Ophthalmol. 2014; 132(8): 985-992. doi: 10.1001/jamaophthalmol.2014.1008.

46. Martin E, Oliver KM, Pearce EI, Tomlinson A, Simmons P, Hagan S. Effect of tear supplements on signs, symptoms and inflammatory markers in dry eye Cytokine. 2018; 105: 37-44. doi: 10.1016/j. cyto.2018.02.009.

47. Boukes RJ, Boonstra A, Breebaart AC, Reits D, Glasius E, Luyendijk L, et al. Analysis of human tear protein profiles using high performance liquid chromatography (HPLC). Doc Ophthalmol. 1987; 67(1-2): 105-113. doi: 10.1007/bf00142704.

48. Srinivasan S, Thangavelu M, Zhang L, Green KB, Nichols KK. iTRAQ quantitative proteomics in the analysis of tears in dry eye patients. Invest Ophthalmol Vis Sci. 2012; 53(8): 5052-5059. doi: 10.1167/iovs.11-9022.

49. Caffery B, Joyce E, Boone A, Slomovic A, Simpson $\mathrm{T}$, Jones L, et al. Tear lipocalin and lysozyme in Sjogren and non-Sjogren dry eye. Optom Vis Sci. 2008; 85(8): 661-667. doi: 10.1097/ OPX.0b013e318181ae4f.

50. Versura P, Nanni P, Bavelloni A, Blalock W, Piazzi M, Roda A, et al. Tear proteomics in evaporative dry eye disease. Eye (Lond). 2010; 24(8): 1396-402. doi: 10.1038/eye.2010.7.

51. Seen S, Tong L. Dry eye disease and oxidative stress. Acta Ophthalmol. 2018; 96(4): e412-e420. doi: 10.1111/aos.13526.

52. Ohashi Y, Dogru M, Tsubota K. Laboratory findings in tear fluid analysis. Clin Chim Acta. 2006; 369(1): 17-28. doi: 10.1016/j.cca.2005.12.035.

53. Mann A, Tighe B. Tear analysis and lens-tear interactions Part I. Protein fingerprinting with microfluidic technology. Cont Lens Anterior Eye. 2007; 30(3): 163-173. doi: 10.1016/j. clae.2007.03.006.

54. Vanarsa K, Ye Y, Han J, Xie C, Mohan C, Wu T. Inflammation associated anemia and ferritin as disease markers in SLE. Arthritis Res Ther. 2012; 14(4): R182. doi: 10.1186/ar4012.

55. Milner MS, Beckman KA, Luchs JI, Allen QB, Awdeh RM, Berdahl J, et al. Dysfunctional tear syndrome: dry eye disease and associated tear film disorders-new strategies for diagnosis and treatment. Curr Opin Ophthalmol. 2017; 27(Suppl 1): 3-47. doi: 10.1097/01.icu.0000512373. 81749.b7.

56. Gao P, Simpson JL, Zhang J, Gibson PG. Galectin-3: its role in asthma and potential as an antiinflammatory target. Respir Res. 2013; 14(1):136. doi: 10.1186/1465-9921-14-136.

57. Puthenedam M, Wu F, Shetye A, Michaels A, Rhee KJ, Kwon JH. Matrilysin-1 (MMP7) cleaves galectin-3 and inhibits wound healing in intestinal epithelial cells. Inflamm Bowel Dis. 2011; 17(1): 260-267. doi: 10.1002/ibd.21443.

58. Menachem-Zidon O, Avital A, Ben-Menahem Y, Goshen I, Kreisel T, Shmueli EM, et al. Astrocytes support hippocampal-dependent memory and long-term potentiation via interleukin-1 signaling. Brain Behav Immun. 2011; 25(5): 1008-1016. doi: 10.1016/j.bbi.2010.11.007.

59. Boehm N, Riechard A, Wiegand M, Pfieffer N. Proinflammatory Cytokine Profiling of tears from dry eye patients by means of antibody microarrays. Invest Ophthalmol Vis Sci. 2011; 52(10): 7725 7730. doi: 10.1167/iovs.11-7266.

60. Hu X, Topouzis S, Liang L, Stotish R. Myostatin signaling through Smad2, Smad3 and Smad4 is regulated by the inhibitory $\operatorname{Smad} 7$ by a negative feedback mechanism. Cytokine. 2004; 26(6): 262272. doi: 10.1016/j.cyto.2004.03.007. 
61. Moudgil KD, Choubey D. Cytokines in autoimmunity: role in induction, regulation, and treatment. J Interferon Cytokine Res. 2011; 31(10): 695-703. doi: 10.1089/jir.2011.0065.

62. Kang MH, Kim MK, Lee HJ, Lee HI, Wee WR, Lee JH. Interleukin-17 in various ocular surface inflammatory diseases. J Korean Med Sci. 2011; 26(7): 938-944. doi: 10.3346/jkms.2011.26.7.938.

63. Kyung-Sun N, Jee-Won M, Ja K, Chang R, Choun-Ki J. Correlations between tear cytokines, chemokines, and soluble receptors and clinical severity of dry eye disease. Invest Ophthalmol Vis Sci. 2012; 53(9): 5443-5450. doi: 10.1167/ iovs.11-9417.

64. Colligris B, Crooke A, Huete-Toral F, Pintor J. An update on dry eye disease molecular treatment: advances in drug pipelines. Expert Opin Pharmacother. 2014; 15(10): 1371-1390. doi: 10.1517/14656566.2014.914492.

65. Albertsmeyer AC, Kakkassery V, Spurr-Michaud S, Beeks O, Gipson IK. Effect of pro-inflammatory mediators on membrane-associated mucins expressed by human ocular surface epithelial cells. Exp Eye Res. 2010; 90(3): 444-451. doi: 10.1016/j. exer.2009.12.009.

66. Coursey TG, de Paiva CS. Managing Sjögren's Syndrome and non-Sjögren Syndrome dry eye with anti-inflammatory therapy. Clin Ophthalmol. 2014; 8: 1447-1458. doi: 10.2147/OPTH.S35685.

67. Pflugfelder S, De Paiva C, Villarreal A, Stern M. Effects of sequential artificial tear and cyclosporine emulsion therapy on conjunctival goblet cell density and transforming growth factor beta2 production. Cornea. 2008; 27(1): 64-69. doi: 10.1097/ ICO.0b013e318158f6dc.

68. D’Souza S, Tong L. Practical issues concerning tear protein assays in dry eye. Eye Vis (Lond). 2014; 1:6. doi: 10.1186/s40662-014-0006-y.

69. Solomon A, Dursun D, Liu Z, Xie Y, Macri A, Pflugfelder S. Pro- and Anti-inflammatory forms of interleukin-1 in the tear fluid and conjunctiva of patients with dry-eye disease. Invest Ophthalmol Vis Sci. 2001; 42(10): 2283-2292.

70. Liu R, Rong B, Tu P, Tang Y, Song W, Toyos R, et al. Analysis of Cytokine levels in tears and clinical correlations after intense pulsed light treating meibomian gland dysfunction. Am J Ophthalmol. 2017; 183: 81-90. doi: 10.1016/j.ajo.2017.08.021.

71. Gipson IK. The ocular surface: the challenge to enable and protect vision: the Friedenwald lecture. Invest Ophthalmol Vis Sci. 2007; 48(10): 43904398. doi: 10.1167/iovs.07-0770.
72. Thornton D, Rousseau K, McGuckin M. Structure and function of the polymeric mucins in airways mucus. Annu Rev Physiol. 2008; 70: 459-486. doi: 10.1146/annurev.physiol.70.113006.100702.

73. Nakamura S, Kinoshita S, Yokoi N, Ogawa Y, Shibuya M, Nakashima $H$, et al. Lacrimal hypofunction as a new mechanism of dry eye in visual display terminal users. PLoS One. 2010; 5(6): e11119. doi: 10.1371/journal.pone.0011119.

74. Lam H, Bleiden L, de Paiva C, Farley W, Stern M, Pflugfelder S. Tear cytokine profiles in dysfunctional tear syndrome. Am J Ophthalmol. 2009; 147(2): 198-205. doi: 10.1016/j.ajo.2008.08.032.

75. Saijyothi A, Angayarkanni N, Syama C, Utpal T, Shweta A, Bhaskar S. et al. Two dimensional electrophoretic analysis of human tears: collection method in dry eye syndrome. Electrophoresis. 2010; 31(20): 3420-3427. doi: 10.1002/elps.201000271.

76. Grus F, Sabuncuo P, Dick H, Augustin A, Pfeiffer $\mathrm{N}$. Changes in the tear proteins of diabetic patients. BMC ophthalmology. 2002; 2:4. doi: 10.1186/14712415-2-4.

77. Böhm D, Keller K, Pieter J, Boehm N, Wolters D, Siggelkow W, et al. Comparison of tear protein levels in breast cancer patients and healthy controls using a de novo proteomic approach. Oncol Rep. 2012; 28(2): 429-438. doi: 10.3892/or.2012.1849. 